\title{
Development of an automatic procedure to estimate the reflection height of tweek atmospherics
}

\author{
Hiroyo Ohya ${ }^{1}$, Kazuo Shiokawa $^{2}$, and Yoshizumi Miyoshi ${ }^{2}$ \\ ${ }^{1}$ Graduate School of Engineering, Chiba University, 1-33 Yayoi-cho, Inage-ku, Chiba 263-8522, Japan \\ ${ }^{2}$ Solar-Terrestrial Environment Laboratory, Nagoya University, Furo-cho, Chikusa-ku, Nagoya 464-8601, Japan
}

(Received February 15, 2008; Revised May 2, 2008; Accepted May 8, 2008; Online published September 8, 2008)

\begin{abstract}
This paper presents an automated procedure to estimate apparent reflection height $h$ (from the cutoff frequency for the first waveguide mode, $f_{\mathrm{c}}$ ), horizontal propagation distance $d$, and propagation time $T_{\mathrm{g}}$ of tweek atmospherics. Tweek data recorded at the Kagoshima Observatory $\left(31.48^{\circ} \mathrm{N}, 130.72^{\circ} \mathrm{E}\right)$, Japan, were used to evaluate the procedure by comparing the results estimated by the automatic method to those read manually by an operator. The two types of results showed differences (automatic-manual) of $+0.58 \mathrm{~km},-9.9 \mathrm{~Hz}$, and $+3058.9 \mathrm{~km}$ for mean $h, f_{\mathrm{c}}$, and $d$, respectively. The difference in $h\left(f_{\mathrm{c}}\right)$ was less than the resolution of the fast Fourier transform used to obtain the tweek spectra. These comparisons indicate that the automatic estimation procedure of tweek parameters developed in this paper performs well and is a useful tool for studying long-term height variations of the ionospheric $D$ and lower $E$ regions using very low frequency (VLF) and extremely low frequency (ELF) records observed in Japan over the past 30 years.
\end{abstract}

Key words: Tweek atmospherics, reflection height, $D$-region ionosphere, low latitudes.

\section{Introduction}

The nighttime $D$-region ionosphere at altitudes of 60 $90 \mathrm{~km}$ is produced from photoionization by geocoronal emissions as well as ionization by radiation belt particles at middle and low latitudes (e.g., Tohmatsu, 1990). Thus, measurements of the nighttime $D$-region at low latitudes can be used to monitor these effects, particularly those of radiation belt particles during geomagnetic storms.

Strong coupling between the $D$-region ionosphere and the neutral atmosphere makes $D$-region measurements difficult. For example, the electron density of the $D$-region is too low to be measured by conventional ionosondes with frequencies of $1-30 \mathrm{MHz}$. The altitude of the $D$-region is too low for satellite measurements of electron density and too high for balloon measurements. Thus, sounding rocket experiments have been used to directly measure the $D$ region electron density profiles (e.g., Maeda, 1971). However, sounding rockets can only be launched from limited locations and obtain limited frequencies. The recent development of medium frequency (MF) radar has enabled continuous measurements of electron density in the $D$-region (e.g., Holdsworth et al., 2002), although drawbacks of MF radars include their need for rather large facilities as well as large amounts of electrical power. Standard radio-wave signals propagated from very low frequency/low frequency (VLF/LF) transmitters have also been used to measure the height variation of the $D$-region, based on phase and intensity modulations (e.g., Bickel et al., 1970; Thomas and Harrison, 1970; Thomson, 1993). However, the region avail-

Copyright (c) The Society of Geomagnetism and Earth, Planetary and Space Sciences (SGEPSS); The Seismological Society of Japan; The Volcanological Society of Japan; The Geodetic Society of Japan; The Japanese Society for Planetary Sciences; TERRAPUB able for electron density measurements by this method is restricted along the propagation path between the transmitter and the receiver.

Ohya et al. (2003) proposed a technique to measure the $D$-region height variations using VLF/extremely low frequency (ELF) tweek atmospherics $(1.5-10.0 \mathrm{kHz})$ originating from lightning discharges. Passive measurement of VLF/ELF tweeks is much easier and cheaper than measurement by active radars. Moreover, since the tweek atmospherics can always be detected, the technique is a powerful tool for deriving the long-term variation of the $D$-region height. The technique is based on the fact that the firstorder mode cut-off frequency of tweek atmospherics is a significant indicator of tweek reflection heights (equivalent electron densities) in the $D$-region ionosphere. Tweeks are reflected by the $D$ - and lower $E$-region ionosphere at altitudes below $100 \mathrm{~km}$ where the electron density becomes 20-28 cm $\mathrm{cm}^{-3}$ (Shvets and Hayakawa, 1998; Ohya et al., 2003) and propagate long distances (a few thousand kilometers) in the Earth-ionosphere waveguide (Outsu, 1960; Yamashita, 1978; Hayakawa et al., 1994, 1995; Shvets and Hayakawa, 1998). As for the polarization of the tweeks, the zero-order mode of tweeks is expected to be $\mathrm{TM}_{0}$ (transverse magnetic) mode (Wait, 1972), in agreement with the observations by Hayakawa et al. (1994). For the first-order mode, both TM and TE (transverse electric) modes were considered by Hayakawa et al. (1994) based on an inhomogeneous and anisotropic ionosphere model. Yamashita (1978) showed by calculating attenuation coefficient and excitation factor of the waveguide near the cut-off frequencies that QTE (quasi-TE) mode plays a dominant role in the formation of tweeks. Ferencz (2004) presented a new theoretical model of waveguide and numerical solutions of 
the model. Ferencz et al. (2007) recently reported by using VLF recordings on board the DEMETER satellite that specific signals of fractional hop whistlers, "Spiky Whistler", are excited by tweeks.

From the cut-off frequency of the first mode of tweeks, the apparent reflection height $h$ can be estimated. Lower (higher) $h$ corresponds to increased (decreased) electron density in the $D$-region ionosphere. Thus, the $h$ value obtained from tweeks is a useful parameter for investigating electron density variations in the nighttime $D$-region ionosphere (e.g., Ohya et al., 2003, 2006). The $h$ denotes the height of the waveguide averaged over the propagation path (Hayakawa et al., 1994). Since a few hundred tweeks are received in 1 minute at night and the various propagation paths of those tweeks can be detected, the tweek method is a powerful tool for investigating the variations in the $D$ region height over a wide area. Moreover, it is possible to investigate the height variations during various geophysical time scales such as during magnetic storms and solar cycles.

In Japan, tweek atmospherics have been observed at the Moshiri $\left(44.37^{\circ} \mathrm{N}, 142.27^{\circ} \mathrm{E}\right)$ and Kagoshima $\left(31.48^{\circ} \mathrm{N}\right.$, $130.72^{\circ} \mathrm{E}$ ) observatories of the Solar-Terrestrial Environment Laboratory, Nagoya University, for more than 30 years. With this 30 -year dataset, analysis of the longterm variations of the $D$-region and lower $E$-region ionosphere is possible. However, for efficient analysis of a longterm dataset, a new method that can detect tweeks and estimate the cut-off frequency automatically is essential. In this study, we developed such a method and verified it by comparing the parameters obtained by automatic and conventional manual methods.

\section{Automatic Method with a Spherical Waveguide Model}

\subsection{Spherical waveguide model}

The routine for automatic estimation of the ionosphere parameter was based on a homogeneous spherical Earthionosphere waveguide model (Davies, 1969). The horizontal phase velocity $v_{\mathrm{p}}$ in the homogeneous spherical Earthionosphere waveguide is approximated by

$$
v_{\mathrm{p}} \approx \frac{c\left(1-\frac{h}{a}\right)}{\sqrt{1-\left(\frac{n \lambda}{2 h}\right)^{2}}}
$$

where $c$ is the light velocity, $h$ is the height of the waveguide, $a$ is the Earth's radius, $\lambda$ is the wavelength in free space, and $n$ is the order of mode. The denominator of Eq. (1) indicates real part of sine of the incident angle to calculate the horizontal group velocity. Equation (2) gives $h$ as

$$
h=\frac{n c}{2 f_{\mathrm{c}}}
$$

where $f_{\mathrm{c}}$ is the cut-off frequency. We do not need to take the number of reflections into account, although multiple reflections in the waveguide are geometrically described by image sources placed along vertical axis at the points, 0 , $\pm 2 h, \pm 4 h, \ldots$ when we assume a vertical dipole for lightning discharge on the ground (Wait, 1972). We estimate the
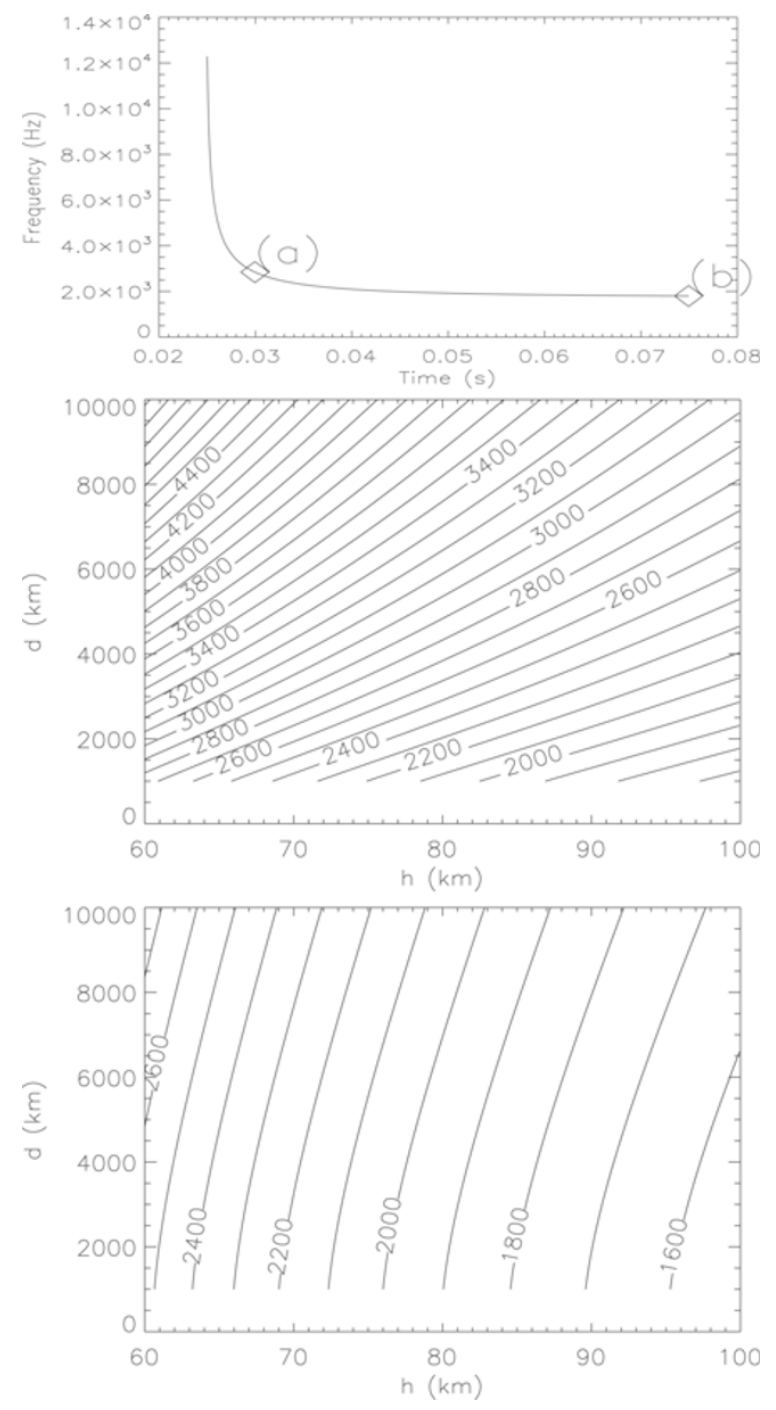

Fig. 1. (Top panel) frequency-time curve of an artificial tweek, (middle panel) dependence of the parameter $h$ and $d$ on the frequency of the tweek at the time of (a), and (bottom panel) dependence of the parameter $h$ and $d$ on the frequency of the tweek at the time of (b). In the middle and bottom panels, the contour lines with the numbers indicate the frequency $f(\mathrm{~Hz})$ of the tweeks.

cut-off frequency for the first-order mode. The cut-off frequency (or cut-off wavelength) is determined by the height of the waveguide. The horizontal group velocity $v_{\mathrm{g}}$ for the first-order mode is obtained from Eqs. (1) and (2) for $n=1$ as follows:

$$
\begin{aligned}
v_{\mathrm{g}} & \approx \frac{c^{2}}{v_{\mathrm{p}}} \\
& =\frac{c \sqrt{1-\left(\frac{f_{\mathrm{c}}}{f}\right)^{2}}}{1-\frac{c}{2 a f_{\mathrm{c}}}}
\end{aligned}
$$

where $f$ is the frequency of the waves. The propagation time $T_{\mathrm{g}}$ for the first-order mode is obtained from Eq. (4):

$$
T_{\mathrm{g}}=\frac{d}{v_{\mathrm{g}}}
$$




$$
=\frac{d\left(1-\frac{c}{2 a f_{\mathrm{c}}}\right)}{c \sqrt{1-\left(\frac{f_{\mathrm{c}}}{f}\right)^{2}}}
$$

where $d$ is the horizontal propagation distance of tweeks. Consequently, the frequency-time dispersion relation of tweeks is rewritten as follows:

$$
f=\frac{T_{\mathrm{g}} f_{\mathrm{c}}}{\left(1-\frac{c}{2 a f_{\mathrm{c}}}\right){\sqrt{T_{\mathrm{g}}^{2}-\left(\frac{d}{c}\right)^{2}}}^{2}}
$$

In Eq. (5), unknown parameters are $f_{\mathrm{c}}, d$, and $T_{\mathrm{g}}$. Here $T_{\mathrm{g}}$ is the time delay from the lightning to the observation point for each frequency. For easier calculation of these unknown parameters, we transformed Eq. (5). We assumed that the unknown parameter $T_{\mathrm{g}}$ is the time from lightning occurrence to the first point $t_{0}$, where $t_{0}$ is the first time the tweek is identified on the dynamic spectrum. Thus, we can use $t_{0}=T_{\mathrm{g}}, t_{1}=T_{\mathrm{g}}+$ time step, $t_{2}=T_{\mathrm{g}}+$ time step $\times 2, \ldots$, and $t_{n}=T_{\mathrm{g}}+$ time step $\times N$. Then, by using the gradient-expansion algorithm, we fitted Eq. (5) to the dataset of $\left(t_{i}, f_{i}\right)$ for $i=0,1,2, \ldots, N$, which was determined from the dynamic spectrum, as described in Section 2.2.

Figure 1 shows dependence of the parameters $h$ and $d$ on the frequency $f$. Top panel shows a typical frequencytime curve of an artificial tweek. Middle panel shows the dependence of the $h$ and $d$ on the $f$ at the time of (a). The $x$, and $y$ axes indicate $h(\mathrm{~km})$ and $d(\mathrm{~km})$, respectively, and the contour indicates $f(\mathrm{~Hz})$. The middle panel shows that $d$ is more sensitive to the frequency changes than $h$ at the time of the maximum curvature. The bottom panel shows the dependences at the time of (b) in the top panel. This panel indicates that $h$ becomes more sensitive to $f$-changes at the tweek tail.

\subsection{Automatic method}

Figure 2 shows an example of tweek atmospherics observed at Kagoshima at 17:51 UT (02:51 LT) on 1 November 2003. A tweek atmospheric was observed at 73.84$73.95 \mathrm{~s}(17: 51: 13.84-17: 51: 13.97 \mathrm{UT})$, as indicated by the two white vertical lines illustrating the analyzed time interval. The frequencies decreased from $4-2 \mathrm{kHz}$ to just below $2 \mathrm{kHz}$, showing a characteristic dispersion. The cut-off frequency of the first-order mode was found below $2 \mathrm{kHz}$, and the frequency below the cut-off frequency corresponded to the zero-order mode. By fitting a theoretical curve to these observed tweeks, the parameters $h, d$, and $T_{\mathrm{g}}$ can be estimated. We adopted the gradient-expansion algorithm to fit the theoretical function of Eq. (5) based on the homogeneous spherical waveguide model.

At the Kagoshima and Moshiri observatories, the waveforms (an east-west magnetic component) of these VLF/ELF atmospherics in a wide frequency range $(0$ $10 \mathrm{kHz}$ ) have been recorded for 2 minutes of every hour (from minutes 50 to 52) since April 1976 onto analog tapes and mini-disks. Because sampling for each entire hour would produce massive amounts of VLF/ELF data, only the 2-min interval per hour has been recorded continuously for more than 30 years. These analog tweek waveforms were digitized into a hard disk with a 16-bit A/D converter with a $20-\mathrm{kHz}$ sampling frequency. The automated procedure first picks up intense tweeks, which have amplitudes exceeding $80 \%$ of the maximum amplitude of the 2 minutes. By this method, the tweek level should vary among the 2-minute datasets. We cannot discuss the intensity variations of VLF data over the long term because the variations depend not only on the condition of the $D$-region and lower $E$-region but also on the observational system itself. On the other hand, the reflection-height variations of tweeks do not de-

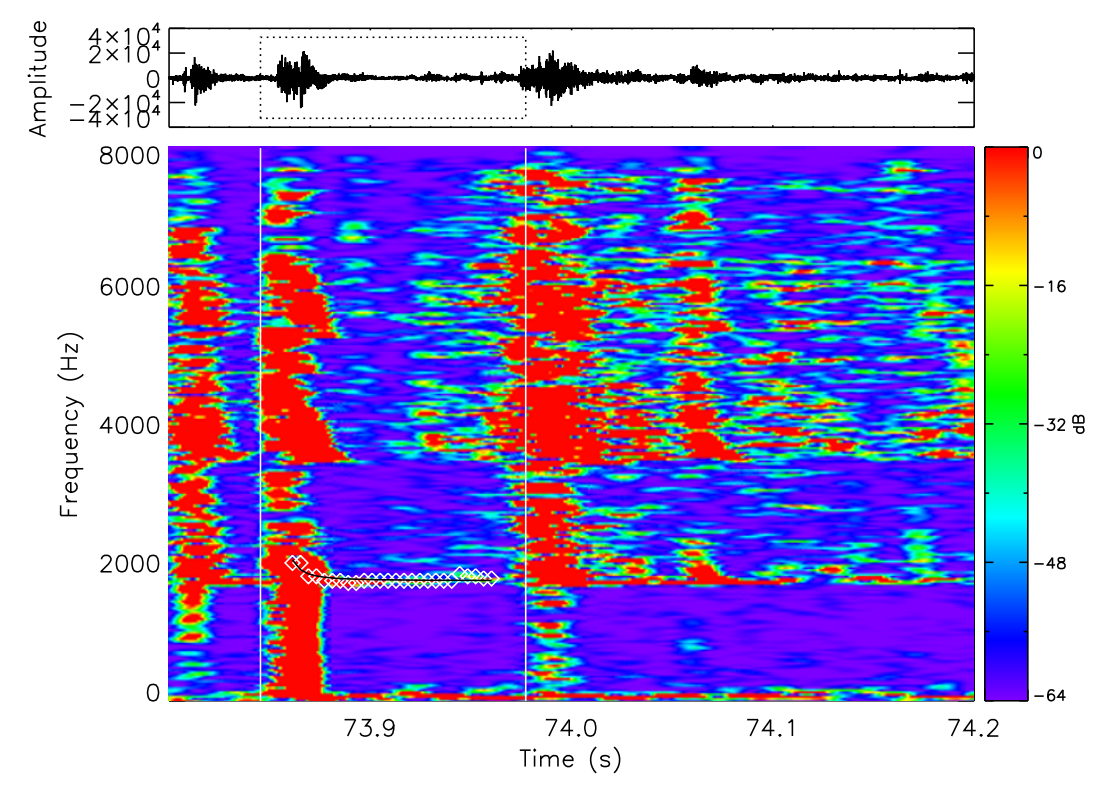

Fig. 2. (Top) waveform and (bottom) dynamic spectrum of a clear tweek atmospheric observed at Kagoshima Observatory at 17:51:13.84-17:51:13.97 UT on 1 November 2003. The white points and black curve indicate the spectral peaks and a theoretical curve determined by the automatic procedure, respectively. 
pend on the observational system and are useful for investigating long-term change.

Next, the procedure searches the frequency $f_{i}$ of the maximum power of the spectrum at every sampling time $t_{i}(50 \mu \mathrm{s})$ for time intervals from $30 \mathrm{~ms}$ before to $70 \mathrm{~ms}$ after the time triggered by shifting the fast Fourier transform (FFT) window (25.6 ms) by one sampling time $t_{i}$ on the dynamic spectrum. The duration of tweeks is typically $\sim 50 \mathrm{~ms}$. Considering that the longest tweeks last $\sim 100 \mathrm{~ms}$, we empirically set the time intervals of $100 \mathrm{~ms}$ to have enough fitting precision and to exclude the next (non-target) tweeks. The first time interval of $30 \mathrm{~ms}$ needs to obtain the higher-frequency part of the tweeks. The last time interval of $70 \mathrm{~ms}$ is long enough to obtain the data points of the tweek tail. Then, to remove background noise emissions and other tweeks embedded on the target tweek, the procedure selects the dataset $\left(t_{i}, f_{i}\right)$ for one tweek automatically by checking the frequency difference between two successive times, $t_{i}$ and $t_{i+1}$. However, if more than two tweeks with comparable intensities overlap, the procedure cannot clearly distinguish them and fails to determine the parameters. Such cases can be finally removed, since the fitting error (expressed as chi-squared below) becomes very large, and the estimated parameter $d$ becomes unrealistic (either less than $1,000 \mathrm{~km}$ or more than $10,000 \mathrm{~km}$ ) for such cases.

For the least-square fitting, we adopted the fitting results when the mean difference between the data point $f_{i}$ and the fitted curve was less than $50 \mathrm{~Hz}$, which is comparable to the FFT frequency resolution $(40 \mathrm{~Hz})$. The fitting results with large differences should thus be ruled out.

\section{Comparison to the Manual Method}

3.1 Evaluation of the developed method using artificial tweeks

To see the difference between the automated and manual method, we first compared the frequency data points de- termined by the automated and manual methods by using waveforms of artificial tweeks. We calculated the frequencies $f$ of the artificial tweek using Eq. (5) and produced the waveform $y(t)=\sin (2 \pi f t)$. The amplitude and initial phase were assumed to be 1 and 0 , respectively.

Figure 3 shows a frequency-time spectra of an artificial tweek with $f_{\mathrm{c}}=1700 \mathrm{~Hz}$ and $d=6000 \mathrm{~km}$. White points and diamonds indicate the data points determined by the automatic procedure and by the manual method, respectively. The solid and dotted curves are the fitting curve by the automatic procedure and the input frequency-time curve, respectively. The results of the estimation were $f_{\mathrm{c}}=1659.18$ $\pm 0.13 \mathrm{~Hz}$ and $d=9408.16 \pm 0.50 \mathrm{~km}$ for the automated method, and $f_{\mathrm{c}}=1712.17 \pm 0.07 \mathrm{~Hz}$ and $d=7125.96 \pm$ $0.07 \mathrm{~km}$ for the manual method, respectively. This means that the automatic procedure can recognize correct peak of the tweek signals.

To investigate the fitting accuracy of the manual method, we generated 9 artificial tweeks that have a parameter combination of $f_{\mathrm{c}}=1500,2000$ and $2500 \mathrm{~Hz}$, and $d=1000$, 6000 and $10000 \mathrm{~km}$. We estimated $f_{\mathrm{c}}$ and $d$ for these artificial tweeks by the manual method and compared the fitting results with original input values. As the results, the average fitting error of the $f_{\mathrm{c}}$ was $+0.716 \%$. The fitting error of the $d$ decreased for longer $d$, i.e., the average errors were $+35.494 \%$ for $d=1000 \mathrm{~km},+18.766 \%$ for $d=6000 \mathrm{~km}$, and $+0.292 \%$ for $d=10000 \mathrm{~km}$, respectively. This means that tweek signals with short duration of frequency-time dispersion are more difficult for estimating $d$.

Next we checked whether the artificial tweeks could be picked up by the automatic procedure according to our tweek selection alogorithm. All tweeks with nonoverlapped time intervals of more than $50 \mathrm{~ms}$ before and after the tweek signals could be picked up by the automatic procedure (100 of 100 tweeks). The automatic procedure did not pick up the tweeks for which other tweeks were

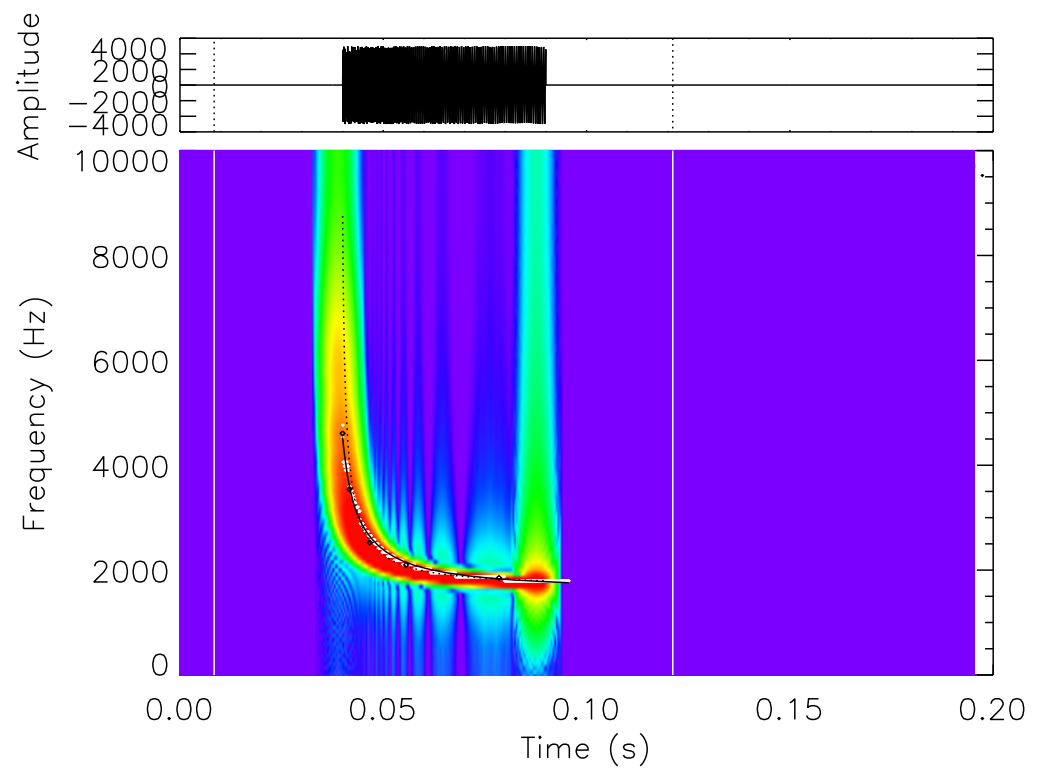

Fig. 3. A frequency-time spectra of an artificial tweek with $f_{\mathrm{c}}=1700 \mathrm{~Hz}$ and $d=6000 \mathrm{~km}$. White points and diamonds indicate the data points determined by the automatic procedure and by the manual method, respectively. The solid and dotted curves are the fitting curve by the automatic procedure and the input frequency-time curve, respectively. 
overlapped during time interval less than $50 \mathrm{~ms}$ before and after the target tweek, because of our tweek-selection criteria.

\subsection{Comparison using observation data}

We compared tweek parameters estimated by the automated and manual methods with the spherical model, using 77 tweeks recorded at Kagoshima at 17:50-17:52 UT (02:50-02:52 LT) on 1 November 2003. The automatic procedure picked up 266 tweeks. For 93 of those 266 tweeks, the typical dispersion relation could not be found by visual inspection in the manual method. For the remaining 173 tweeks, 96 had a large fitting error $(>50 \mathrm{~Hz})$. These large-error events were mainly caused by the overlapping of more than two tweeks on the dynamic spectrum, as will be discussed later. Thus, we used the other 77 tweeks for the comparison. For each tweek, we compared the automatic estimation results of $h\left(f_{\mathrm{c}}\right)$ and $d$ to those from the manual method, in which an operator selects $\left(t_{i}, f_{i}\right)$ based on visual inspection of the dynamic spectrum and calculates the parameters using Eq. (5).

Figure 2 shows an example of good fitting by the automatic method (the mean difference between the data point $f_{i}$ and the fitted theoretical curve is $42.0 \mathrm{~Hz}$ ). The target tweek was observed at 73.84-73.95 s at frequencies of $\sim 2 \mathrm{kHz}$, as shown by the two white vertical lines indicating the analyzed time interval. The white points and the black line mark the peak frequencies of the tweek and the estimated frequency-time curve, respectively. In this case, the average and the fitting error of the automatically estimated $h\left(f_{\mathrm{c}}\right)$ are $89.43 \pm 0.33 \mathrm{~km}(1676.1 \pm 6.2 \mathrm{~Hz})$, while those by the manual method are $89.04 \pm 0.63 \mathrm{~km}(1683.4 \pm 11.8 \mathrm{~Hz})$. The difference in $h$ between the two methods is $0.39 \mathrm{~km}$ $(-7.2 \mathrm{~Hz})$, indicating that quality of the automatic method is comparable to that of the manual method.

Estimations of $d$ are $2022.0 \pm 76.8 \mathrm{~km}$ by the automatic method and $789.1 \pm 14.4 \mathrm{~km}$ by the manual method. The difference of $1232.9 \mathrm{~km}$ for $d$ is much larger than the fitting errors. The value of $d$ depends on the curvature (frequency dispersion) of the tweeks on the dynamic spectrum. Since the frequency falls quite rapidly to $f_{\mathrm{c}}$, it is difficult to obtain sufficient data points at higher frequencies to determine the curvature of the dispersion. The duration of tweeks $(\sim 100 \mathrm{~ms})$ is also not very long compared to the time resolution of the FFT window $(25.6 \mathrm{~ms})$. For these reasons it is difficult to accurately estimate $d$ using the curvature of the tweek spectrum. However, it is necessary to estimate $d$ in higher accuracy when we make distribution maps of both the reflection heights and lightning locations. It is useful to investigate the $D$-region phenomena with such distribution maps, for example, for gamma ray bursts, magnetic storms, earthquakes, and Elves. Thus, estimating $d$ with high accuracy is our future work.

Christian et al. (2003) showed that $78 \%$ of lightning on the Earth occurs between latitudes of $30^{\circ} \mathrm{S}$ and $30^{\circ} \mathrm{N}$, while Lynn and Crouchley (1967) reported that the number of tweeks propagating from the east was about four times larger than the number propagating from the west at Brisbane on the eastern coast of Australia. Interestingly, the rate of lightning occurrence was highest at the west coast of Australia (Christian et al., 2003). Such directionality has been explained by the smaller attenuation coefficient (by about $3 \mathrm{~dB} / 1000 \mathrm{~km}$ ) over sea than over land (Davies, 1969). Considering these previous findings, most tweeks observed at Kagoshima $\left(31.48^{\circ} \mathrm{N}\right)$ probably came from over the sea at lower latitudes.

As noted above, the main reason for erroneous fitting was the overlap of more than two tweeks on the dynamic spectrum. The automated procedure cannot separate overlapping tweeks. Figure 4 presents an example of a failed estimation, in which the fitting trace extends to the spectra of a few tweeks. In this case, the automatic estimation failed, while the manual method still gave reasonable val-

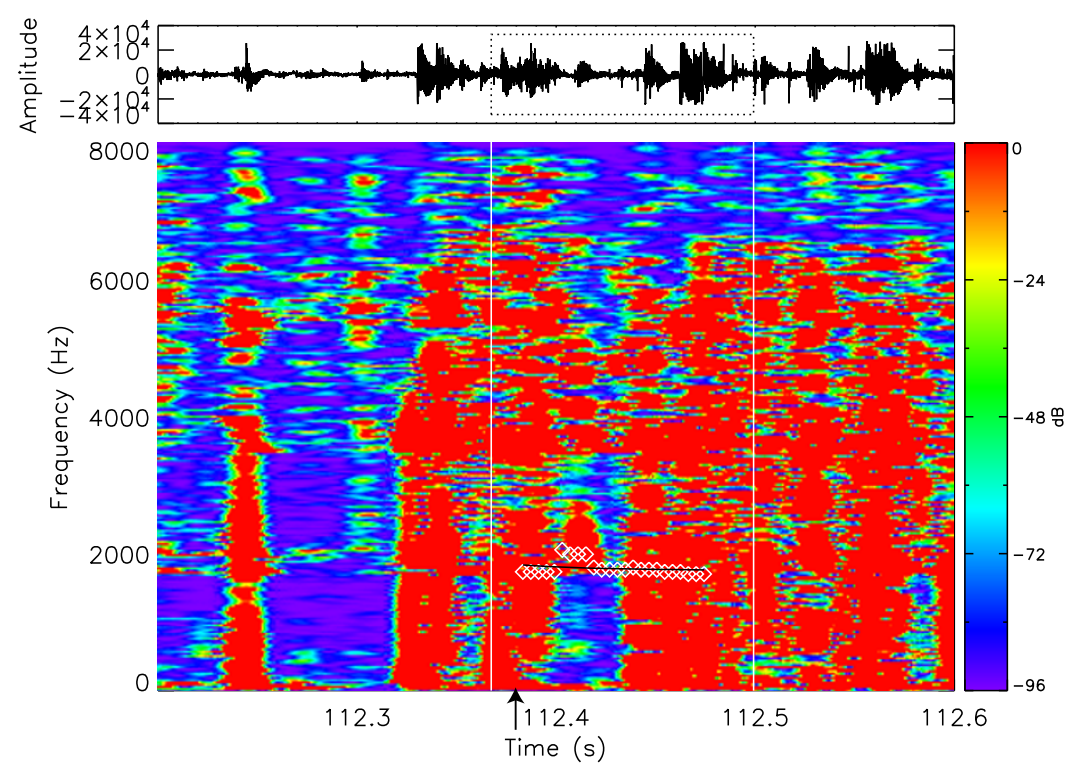

Fig. 4. (Top) waveform and (bottom) dynamic spectrum of multiple overlapped tweek atmospherics observed at Kagoshima Observatory at 17:51:52.37-17:51:52.50 UT on 1 November 2003. The white points and black curve indicate the spectral peaks and a theoretical curve determined by the automatic procedure, respectively. The automatic procedure fails to distinguish the multiple tweeks. 


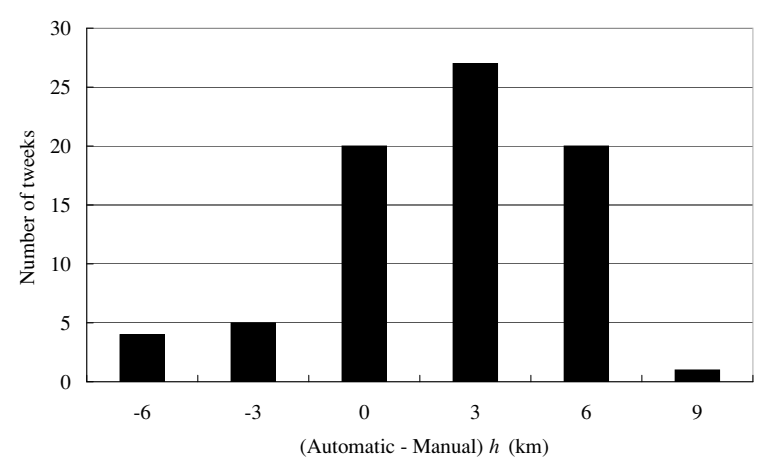

Fig. 5. Differences $\delta$ (= automatic - manual) of the reflection height $h$. Of the total tweeks, $61.0 \%$ fall in the range of $-3 \mathrm{~km}<\delta<3 \mathrm{~km}$.

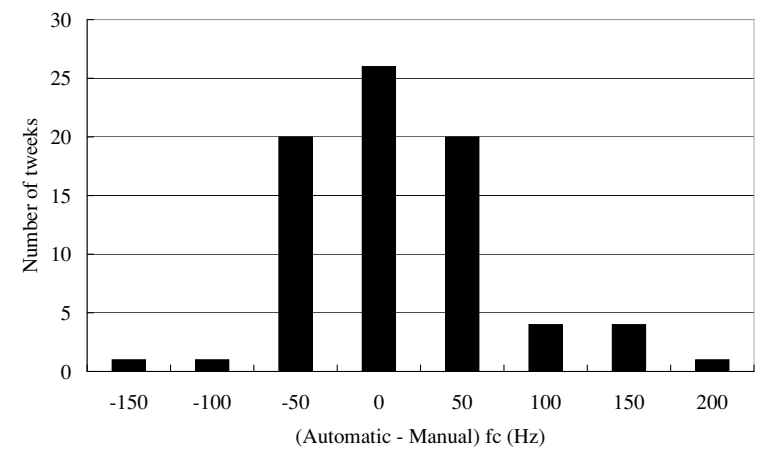

Fig. 6. Differences $\delta$ (= automatic - manual) of the cut-off frequency for the first-order mode $f_{\mathrm{c}}$. Of the total tweeks, $59.7 \%$ fall in the range of $-50 \mathrm{~Hz}<\delta<50 \mathrm{~Hz}$.

ues of $h\left(f_{\mathrm{c}}\right)$ and $d$ of $88.83 \pm 0.24 \mathrm{~km}(1687.5 \pm 9.0 \mathrm{~Hz})$ and $2816.0 \pm 39.0 \mathrm{~km}$, respectively. In this case, the automatic procedure could not separate the target tweek (marked by the black arrow) from several other tweeks, incorrectly recognizing all as one tweek. Such errors caused by overlapping tweeks occurred in approximately $15 \%$ of the total cases. For $72 \%$ of these error cases, $d$ was less than $1000 \mathrm{~km}$, which is smaller than typical values of tweek $d$ values. Thus, by checking the values of $d$, we may be able to eliminate errors caused by overlapping tweeks.

Figures 5, 6, and 7 show the comparison results for $h$, $f_{\mathrm{c}}$, and $d$, respectively, between the automated and manual methods. The value of the $x$-axis indicates the maximum of the interval; in Fig. 5, for example, 0 is the maximum of the interval of -3 to $0 \mathrm{~km}$. Out of 77 cases, acceptable values of $h(-3 \mathrm{~km}<\delta<3 \mathrm{~km}, \delta=$ automatic - manual $)$ and $f_{\mathrm{c}}$ $(-50 \mathrm{~Hz}<\delta<50 \mathrm{~Hz})$ were obtained for $61.0 \%$ and $59.7 \%$, respectively. We considered $f_{\mathrm{c}}(h)$ to be acceptable if the difference was less than $\pm 50 \mathrm{~Hz}( \pm 3.0 \mathrm{~km}$ at $\sim 1600 \mathrm{~Hz})$, considering the spectral resolution of FFT $(40 \mathrm{~Hz})$. On the other hand, in Fig. 7, only $15.6 \%$ of the events have a difference in $d$ of less than $1000 \mathrm{~km}$.

Comparing the results of the automated (for 194 tweeks, including 93 tweeks for which the manual method could not estimate parameters) and manual (for 77 tweeks) methods for the 2-minute averages, the averages and standard deviations of $h, f_{\mathrm{c}}$, and $d$ by the automatic method (manual method) are $89.78 \pm 3.60 \mathrm{~km}$ $(89.20 \pm 2.68 \mathrm{~km}), \quad 1672.2 \pm 68.5 \mathrm{~Hz}(1682.1 \pm 51.8 \mathrm{~Hz})$,

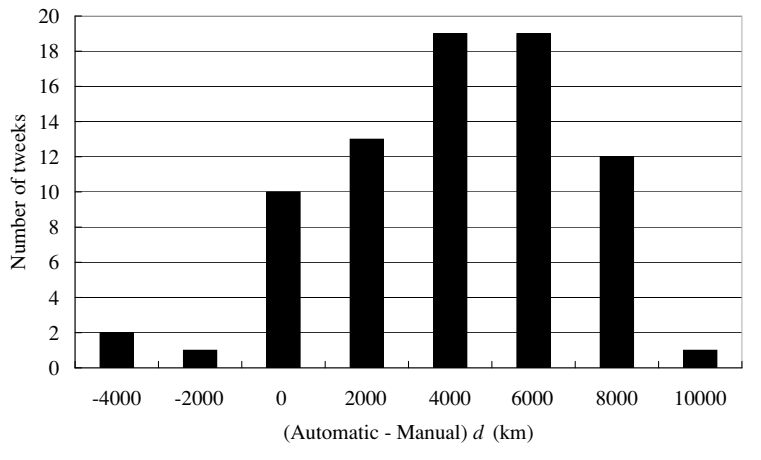

Fig. 7. Differences $\delta$ (= automatic - manual) in the propagation distance $d$. Of the total tweeks, $29.9 \%$ fall in the range of $-2000 \mathrm{~km}<\delta<$ $2000 \mathrm{~km}$.

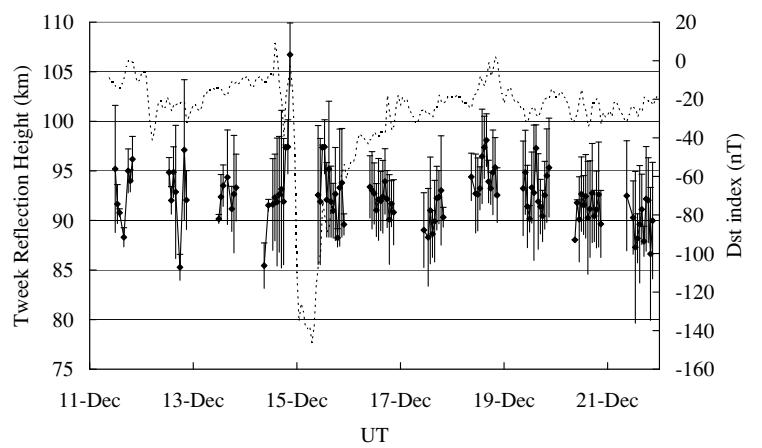

Fig. 8. Reflection height variations of tweeks estimated by the automatic procedure during a magnetic storm of 11-21 December 2006. The diamonds with error bars indicate 2-minute averages and standard deviations of the reflection height. The dotted line indicates the variations of the $D_{\text {st }}$ index.

and $5429.9 \pm 2899.2 \mathrm{~km}(2371.0 \pm 1388.5 \mathrm{~km})$, respectively. Thus, the differences (automatic - manual) between the mean $h, f_{\mathrm{c}}$, and $d$ values are $+0.58 \mathrm{~km},-9.9 \mathrm{~Hz}$, and $+3058.9 \mathrm{~km}$, respectively. The difference for $h\left(f_{\mathrm{c}}\right)$ is less than the resolution of the FFT $(40 \mathrm{~Hz})$, but the difference in $d$ is very large and must be improved.

Figure 8 shows variations of tweek reflection heights obtained by the automatic procedure during an intense magnetic storm on 11-21 December 2006. The plotted data are averages and standard deviations for 2-minute intervals obtained for each hour during nighttime. The total number of tweeks during the plotted interval was 918. For such a large number of tweeks, the automatic procedure is effective in reducing the analysis time. The reflection heights abruptly rose in the initial phase (17:50-20:50 UT, 14 December) (from $91.90 \mathrm{~km}$ to $106.72 \mathrm{~km}$ ) and the recovery phase (9:50-12:50 UT, 15 December) (from $91.90 \mathrm{~km}$ to $97.43 \mathrm{~km}$ ) of the storm. These increases in reflection height occur in response to the characteristic $D_{\text {st }}$ variations, and may indicate temporal decrease of energetic electron fluxes in the inner radiation belt.

\section{Conclusions}

We have developed a procedure for automatic estimation of the reflection height $h$ (cut-off frequency of the first-order mode, $f_{\mathrm{c}}$ ), the propagation distance $d$, and the propagation time $T_{\mathrm{g}}$ of tweek atmospherics. In the case studied here, 
the differences of each ionospheric parameter between the automated and manual methods were $+0.58 \mathrm{~km},-9.9 \mathrm{~Hz}$, and $+3058.9 \mathrm{~km}$ for mean $h, f_{\mathrm{c}}$, and $d$, respectively. The differences in $h\left(f_{\mathrm{c}}\right)$ were sufficiently small and less than the resolution of the FFT. These comparison results indicate that the developed procedure is a reliable method for automatically estimating tweek parameters and can be used to investigate long-term height variations of the $D$-region and lower $E$-region of the ionosphere.

Acknowledgments. We are grateful to late K. Hidaka of the Kagoshima Observatory of the Solar-Terrestrial Environment Laboratory (STEL), Nagoya University, and M. Satoh, Y. Katoh, Y. Hamaguchi, and Y. Yamamoto of STEL for their technical support of the continuous VLF/ELF measurements. This work was supported by Project 2 and the cooperative research program of STEL.

\section{References}

Bickel, J. E., J. A. Ferguson, and G. V. Stanley, Experimental observation of magnetic field effects on VLF propagation at night, Radio Sci., 5, 19, 1970.

Christian, H. J., R. J. Blakeslee, D. J. Bocchippio, W. L. Boeck, D. E. Buechler, K. T. Driscoll, S. J. Goodman, J. M. Hall, W. J. Koshak, D. M. Mach, and M. F. Stewart, Global frequency and distribution of lightnings as observed from space by the Optical Transient Detector, $J$. Geophys. Res., 108, ACL4-1-ACL4-15, 2003.

Davies, K., Ionospheric Radio Waves, Waltham, MA: Blaisdell, 1969.

Ferencz, O. E., Short impulse propagation in waveguides, Hiradastechnika “Communications", LIX, 6, 2-6, 2004.

Ferencz, O. E., Cs. Ferencz, P. Steinbach, J. Lichtenberger, D. Hamar, M. Parrot, F. Lefeuvre, and J.-J. Berthelier, The effect of subionospheric propagation on whistlers recorded by the DEMETER satelliteobservation and modeling, Ann. Geophys., 25, 1103-1112, 2007.

Hayakawa, M., K. Ohta, and K. Baba, Wave characteristics of tweek atmo- spherics deduced from the direction-finding measurement and theoretical interpretation, J. Geophys. Res., 99, 10733-10743, 1994.

Hayakawa, M., K. Ohta, S. Shimakura, and K. Baba, Recent findings on VLF/ELF sferics, J. Atmos. Terr. Phys., 57, 467-477, 1995.

Holdsworth, D. A., R. Vuthaluru, I. M. Reid, and R. A. Vincent, Differential absorption measurements of mesospheric and lower thermospheric electron densities using the Buckland Park MF radar, J. Atmos. Terr. Phys., 64, 2029-2042, 2002.

Lynn, K. J. and J. Crouchley, Night-time sferic propagation at frequencies below 10 kHz, Aust. J. Phys., 20, 101-108, 1967.

Maeda, K.-I., Study on electron density profile in the lower ionosphere, $J$. Geomag. Geoelectr., 23, 133-159, 1971.

Ohya, H., M. Nishino, Y. Murayama, and K. Igarashi, Equivalent electron densities at reflection heights of tweek atmospherics in the low-middle latitude D-region ionosphere, Earth Planets Space, 55, 627-635, 2003.

Ohya, H., M. Nishino, Y. Murayama, K. Igarashi, and A. Saito, Using tweek atmospherics to measure the response of the low-middle latitude $D$-region ionosphere to a magnetic storm, J. Atmos. Solar-Terr. Phys., 68, 697-709, 2006.

Outsu, J., Numerical study of tweeks based on waveguide mode theory, Proc. Res. Inst. Atmos., Nagoya University, 7, 58-71, 1960.

Shvets, A. V. and M. Hayakawa, Polarization effects for tweek propagation, J. Atmos. Solar-Terr. Phys., 60, 461-469, 1998.

Thomas, L. and M. D. Harrison, The electron density distributions in the $D$-region during the night and pre-sunrise period, J. Atmos. Terr. Phys., 32, 1-14, 1970.

Thomson, N. R., Experimental daytime VLF ionospheric parameters, $J$. Atmos. Terr. Phys., 55, 173-184, 1993.

Tohmatsu, T., Compendium of Aeronomy (translated and revised by $T$. Ogawa), Terra Scientific Pub. and Kluwer, 1990.

Yamashita, M., Propagation of tweek atmospherics, J. Atmos. Terr. Physics, 40, 151-153, 1978.

Wait, J. R., Electromagnetic Waves in Stratified Media, Pergamon, New York, 1972.

H. Ohya (e-mail: ohya@faculty.chiba-u.jp), K. Shiokawa, and Y. Miyoshi 